\title{
Autonomic cardiac and cardiorespiratory responses in volleyball athletes compared to recreationally trained individuals
}

\section{Respostas autonômicas cardíacas e cardiorespiratória de atletas de volleyball comparados a indivíduos treinado recreacionalmente}

Alex Souto Maior ${ }^{1}$, Pedro Menezes², Steven Fleck ${ }^{3}$, Travis Bunker ${ }^{4}$, Matthew Rhea ${ }^{4}$, Richard Diego Leite ${ }^{5}$, Roberto Simão

\begin{abstract}
Objective: The aim of this study was to investigate the autonomic cardiac modulation between professional volleyball players (VP) and recreational aerobically trained individuals (RAT). Methods: Twentyeight men were divided into two groups, (VP; $n=14)$ and $(R A T ; n=14)$ and each group was subjected to a sub-maximal progressive ramp treadmill test. Cardiac cycle beat to beat (RR) intervals, was obtained through electrocardiogram recording with the test being stopped when the individual reached $85 \%$ of their maximum age-predicted heart rate (HR). The heart rate variables were calculated from ECG using MATLABbased algorithms. Results: At rest, RAT showed lower values of the mean value of RR interval, the root mean square successive difference of all RR intervals (r-MSSD), low frequency (LF) and total power spectral (TP) when compared to VP. No significant difference between groups was noted for high frequency (HF) and index LF/HF. After exercise, no significant differences were measured between groups. Mean values of $\mathrm{VO}_{2}, \mathrm{~V}_{\mathrm{E}} / \mathrm{VO} 2$, and $\mathrm{V}_{\mathrm{E}}$ showed no significant difference at rest and post effort. Conclusion: The variables such as frequency, intensity and volume may to interfere the autonomic cardiac modulation and possibly indicating a superior protection against exercise-induced ventricular arrhythmias and lower risk of sudden cardiac deaths.
\end{abstract}

Key words: Autonomic System, Aerobic Exercise. High Intense Training.

\section{RESUMO}

Objetivo: O objetivo do presente estudo foi investigar o perfil da modulação autonômica cardíaca entre jogadores profissionais de voleibol (VP) e indivíduos aerobicamente treinados recreativas (RAT).

1. University Augusto Motta (UNISUAM). Master Program in Rehabilitation Sciences, Rio de Janeiro, Brazil.

2. Universidade Federal do Rio de Janeiro. Physical Education Post-Graduation Program. Rio de Janeiro, RJ - Brazil.

3. Sport Science Department. Colorado College. Colorado Springs, Colorado, USA.

4. RACE Rx Academy of Exercise Sciences, Logan, Utah, USA.

5. Universidade Federal do Maranhão. Physical Education Department. São Luis, MA - Brazil.

\author{
Corresponding: \\ Alex Souto Maior \\ University Augusto Motta - UNISUAM. \\ Master Program in Rehabilitation Sciences \\ Praça das Nações, 34 - Bonsucesso. \\ Code 21041010 - Rio de Janeiro, RJ - Brasil
}

Artigo recebido em 08/09/2014 Aprovado para publicação em 24/04/2015 


\begin{abstract}
Métodos: Vinte e oito homens foram divididos em dois grupos (VP; $n=14$ ) e (RAT, $n=14)$. Cada grupo foi submetido a um teste ergométrico sub-máximo. O teste foi interrompido quando o indivíduo atingiu $85 \%$ da frequência cardíaca máxima baseada na idade (HR). Os intervalos R-R foram obtidos através da derivação V5 do eletrocardiograma de 12 derivações. As variáveis de domínio de tempo e frequência da variabilidade da freqüência cardíaca foram calculados utilizando algoritmos baseados em MATLAB. Resultados: Em repouso, RAT apresentaram valores signficativamente reduzidos de intervalo RR, RMSSD, potência espectral de baixa freqüência (LF) e potência espectral total (PT) em relação ao VP. Não houve diferença significativa inter-grupos para potência espectral de alta freqüência (HF) e índice LF/HF. Após o exercício, não foram observadas diferenças significativas inter-grupos. Os valores médios de $\mathrm{VO}_{2}$, VE $/ \mathrm{VO}_{2}$ e VE não apresentaram diferença significativa em repouso e pós esforço inter-grupos. Conclusão: As variáveis como a freqüência, intensidade e volume de treinamento pode interferir na modulação autonômica cardíaca e, possivelmente, indicando uma proteção contra arritmias ventriculares induzidas pelo exercício e menor risco de morte súbita cardíaca.
\end{abstract}

Palavras-chave: Sistema Autonômico. Exercícios Aeróbios. Treinamento de Alta Intensidade.

\section{Introduction}

The analysis of heart rate variability (HRV) in time and frequency domains provides a noninvasive method to establishing the autonomic regulation of heart rate (HR), while identifying the variations between consecutive heart beats. ${ }^{1,2,3}$ The electrocardiogram (ECG) is composed of three sections; the $\mathrm{P}$ wave, QRS wave and $T$ wave. These waves represent the depolarization of the atria, depolarization of the ventricle and repolarization of the ventricle, respectively. Thus, cardiac beat to beat, or RR intervals, is obtained as differences between successive R-wave occurrences in the ECG and represent beat to beat of the heart over time. ${ }^{4}$

The rhythm of the HR is controlled by the sinoatrial (SA) node, which is modulated by both the sympathetic and parasympathetic branches of the autonomic nervous system. ${ }^{5,6,7}$ Sympathetic activity tends to increase HR but the response is slow, typically 2-3 seconds. ${ }^{3,6,8,9}$ Parasympathetic activity, on the other hand, tends to decrease HR but mediates faster (0.2-0.6 seconds). ${ }^{6,10,11}$ Analysis of heart rate variability (HRV) has been used as a tool for noninvasive assessment of cardiac autonomic balance in physiological and pathological conditions. ${ }^{2,3,7,8}$ The time-domain methods of HRV are the simplest to perform because they are applied straight to the series of successive RR interval values. The most evidence of such a measure is the mean value of RR intervals or correspondingly, the mean HR. ${ }^{6,7,12}$ The standard deviation of the normal to normal HR beats (SDNN) and the square root of variance can show short-term as well as long-term RR interval variations. The
RMSSD is difference between successive RR intervals to provide an index of cardiac vagal control. The number of adjacent RR intervals differing more than $50 \mathrm{~ms}$, expressed as a percentage of all intervals over the collection period (pNN50). Power spectral density (PSD) analysis, describes how the variance of the data is distributed over the frequency components into which may be decomposed. ${ }^{12}$ In the frequency-domain method, a PSD estimate is calculated for the RR interval series. ${ }^{12,13}$ The regular PSD estimators implicitly assume equidistant sampling, and the RR interval series are converted to equidistantly sampled series by interpolation methods prior to PSD estimation. ${ }^{12}$

Decrease of HRV is related to the elevated risk of sudden cardiac death and associated with numerous other cardiac events such as heart disease, heart failure, diabetes and hypertension. ${ }^{12}$ In these cases, increased sympathetic drive is associated with arrhythmia and sudden death. ${ }^{1,7,14,15,16}$ For the last two decades high maximal oxygen uptake $\left(\mathrm{VO}_{2 \max }\right)$ has been associated with better autonomic control of the cardiovascular system in athletes compared to sedentary individuals. ${ }^{7,10,17-21}$ Previous studies also suggest that modulation of HR may be influenced by certain physiological factors such as aging, body fatness and specific types of training including aerobic and anaerobic training 9,17,22-25. However, the optimal exercise prescription to change measures of HRV in terms of intensity, stimulus mode and duration remains undefined. $1,7,16,17,18,22,25,26$ Moreover, the fact that endurancetrained individuals have consistently higher HRV than untrained individuals suggests that vigorous training programs are necessary to induce changes in $\mathrm{HRV}^{7,9,13,16,17 .}$ 
In fact, a number of studies have reported conflicting results when using spectral analysis to evaluate HRV during exercise and recovery. ${ }^{8,17,18,22,24}$ Notwithstanding this evidence, most longitudinal studies do not report significant correlations between changes in autonomic control of the cardiovascular system and changes in $\mathrm{VO}_{2 \max }$ induced by aerobic training. ${ }^{9}, 20,22,24,27$ Cross-sectional studies exhibit differences between athletes and sedentary individuals (control), demonstrating a higher increase in parasympathetic tone, followed by a decrease in sympathetic drive in athletes..$^{2,8,9,16,17,24}$ In these reported cases, the resting bradycardia observed is commonly associated with augmented markers of cardiac vagal modulation. ${ }^{20,25}$ There is abundant evidence from cross-sectional studies that trained individuals have higher HRV than untrained individuals. ${ }^{3,10}$ The results from longitudinal studies are equivocal with some showing increased HRV after training $9,19,27$ and others showing no differences. ${ }^{12,25,28,29}$ The model of the training programs might be one of the factors responsible for the variability of the results. ${ }^{18,20,22}$

In most cross-sectional studies the relationship between $\mathrm{VO}_{2 \max }$ and HRV has been investigated regardless of the individual training loads, and the specific stimulus of aerobic and anaerobic training. ${ }^{10,18,19,20,21,26}$ Nevertheless, early studies have been conducted comparing the intensity of training between athletes subjected to aerobic/anaerobic exercise and recreationally aerobic trained individuals. ${ }^{18,20,31} \mathrm{Em}$ pirical and practical knowledge emerging from studies on training-related issues in volleyball such as aerobic and anaerobic profile, strength, agility, speed and plyometrics should be integrated and applied when planning annual training programs for volleyball players. Traditionally, athletes make use of periodization to improve the attributes related to their sport modality. This training method is applied in three phases: preparation- acquisition of athletic fitness; competition-maintenance of athletic fitness and competition peaking; and transition-detraining or active recovery. The major characteristic of the periodization involves the enhancement of body mass, fat-free mass, aerobic profile, strength, agility and speed. These skills are evidently integrated and applied when planning annual training programs especially for volleyball players. ${ }^{32}$

In addition to exercise, other factors influence the behavior of the cardiac autonomic system. Perhaps the most studied of these is lung ventilation, which displays a complex interaction with HR through a mechanism predominantly vagal in nature, termed respiratory sinus arrhythmia (RSA). ${ }^{15}$ When using power spectral analysis to interpret RR interval data, several confounding factors should be considered. It is already known that the higher the volume of air inspired before an apnea, the lower the HR values reached after the initial acceleration of exercise, which emphasizes the magnitude of the biphasic response of the HR frequency during breathing. Brown et al. ${ }^{23}$ studied the power spectrum of nine healthy individuals breathing at seven frequencies and two tidal volumes. They concluded that both respiratory rate as well tidal volume strongly influenced total power spectral (TP), LF and HF. TP was highest at lower breathing frequencies (6-10 breaths/min) and it decreased when the frequency was increased above 10 breaths/min[23]. In this respect, the measurement of breath rate can demonstrate a more effective relationship between breathing and modulation in HR. In comparing these two categories of individuals, volleyball players (VP) and recreationally aerobic trained individuals (RAT), this study aims to investigate if volleyball skills are relevant to improve cardiac autonomic control compared to recreational aerobically trained individuals.

\section{Methods}

\section{Experimental Approach to the Problem}

Before the experimental program, 28 men were assigned to 1 of 2 groups: recreationally aerobic trained individuals (RAT; $n=14$ ) and professional volleyball players (VP; $\mathrm{n}=14$ ). Both groups were submitted to sub-maximal progressive ramp treadmill testing under the supervision of experienced fitness professionals. The RR intervals were continuously monitored in supine position for $10 \mathrm{~min}$ at rest, and after exercise using a 12-lead ECG monitor system. The test was stopped when the individual reached $85 \%$ of their maximum age-predicted HR.

\section{Subjects}

Twenty eight healthy male subjects were selected and divided into two groups: VP (athletes: $\mathrm{n}=$ $14 ; 23.5 \pm 5.3$ years, $75.2 \pm 8.2 \mathrm{~kg}, 185.6 \pm 10.2 \mathrm{~cm}$, $23.5 \pm 4.5 \mathrm{~kg} \cdot \mathrm{m}^{-2}$ ) and RAT (recreationally aerobic trained individuals: $\mathrm{n}=14 ; 24.2 \pm 4.2$ years, $76.6 \pm$ $23.9 \mathrm{~kg}, 178 \pm 10 \mathrm{~cm}, 22.5 \pm 5.4 \mathrm{~kg} \cdot \mathrm{m}^{-2}$ ). Individuals 
were informed of the procedures to be performed in two non-consecutive visits to the laboratory ( $\neq 24 \mathrm{~h}$ ). All subjects were considered healthy on the basis of history, anthropometric values (height, body mass and body mass index), hemodynamic measurements (resting blood pressure), and normal resting electrocardiogram (ECG). ${ }^{33}$ All subjects were nonsmokers, nonalcohol users, non-illicit drug users (cocaine, marijuana, and heroine), nonusers of psychotropic drugs, and asymptomatic for cardiovascular, respiratory, and metabolic disease.

Exclusion criteria were refusal to participate in the research, atrial fibrillation, significant valvular heart disease, coronary artery disease, systemic hypertension (blood pressure higher than $140 \times 90$ $\mathrm{mmHg}$ or the use of antihypertensive medication), and metabolic disease.

An inclusion criterion was consistent participation in running activity for more than 12 months prior [34]. Additional criteria: (i) for RAT: individuals filled out an application in order to inform the researchers of their running routine and the subjects who were selected had to join at least one of the official $10 \mathrm{~km}$ runs in Rio de Janeiro. The same individuals reported a running program of minimum 3 times per week with effort perception moderate/strong (intensity of $65 \%$ - 85\% age-zone training, for 30-40 min;); (ii) for VP: subjects were a member of the University's volleyball team as well as participating in Rio de Janeiro Professional Volleyball League. Subjects began their participation in the present study one week after the conclusion of competition period, which consisted of 3 months of pre-season training (4 times/ week of volleyball skills and aerobic/anaerobic stimuli training) followed by 4 months of competition ( 2 matches/week; 3 times/week volleyball skills and aerobic stimuli training). The experimental protocol was in accordance with the declaration of Helsinki and the study protocol was approved by the Institutional Review Board.

\section{Submaximal Exercise Testing}

All testing was performed between 1:00 and 3:00 PM. Subjects received a light lunch $2 \mathrm{~h}$ before the test. Coffee, tea and alcohol intake was prohibited for $12 \mathrm{~h}$ and subjects avoided formal and strenuous exercise for $48 \mathrm{~h}$ before testing. Tests were performed on a motor-driven treadmill (Inbramed 10200, Brazil) using a ramp protocol, wherein the rate of increment in speed and inclination was determined in- dividually by the subjects, with the goal of reaching volitional exhaustion in approximately 8 to $12 \mathrm{~min}$. The test was preceded by a 3-min warm-up followed by incremental increases in speed and incline at every 1 -minute stage. The test was stopped when the individual reached $85 \%$ of their maximum age-predicted heart rate (HR). Subjects were allowed sufficient practice during preliminary testing to become familiar with the treadmill. Ambient air temperature was $22^{\circ} \mathrm{C}$ to $24^{\circ} \mathrm{C}$.

Testing was symptom limited and was terminated if subjects reported dyspnea, chest pain, or any medical reasons including; horizontal or down-sloping ST-segment depression of $\geq 1 \mathrm{~mm}$, ST segment elevation $>1 \mathrm{~mm}$ in non-Q wave lead, atrial fibrillation or supraventricular tachycardia (suggestive of the left bundle branch block), abnormally elevated blood pressure response (blood pressure $\geq 220 \times 120$ $\mathrm{mmHg}$ ), fall in systolic blood pressure $(>20 \mathrm{~mm} \mathrm{Hg}$ ), variation in diastolic pressure under stress greater than $15 \mathrm{mmHg}$, presyncope, severe arrhythmias, presence of extrasystoles, ataxia or ventricular ectopy (presence of 6 or more premature ventricular beats per minute in recovery) and development of bundlebranch block or Intraventricular Conduction Delay (IVCD) that cannot be distinguished from ventricular tachycardia. ${ }^{14}$

\section{Respired gas analysis}

The measurement of gas exchange is an important tool for assessing the cardiac risk and aerobic functional capacity in submaximal exercise testing. The respired gas analysis began by assessing the subjects at rest and post effort for five minutes with the FitMate-Pro $^{\mathrm{TM}}$ (Cosmed, Rome - Italy). Measurements of oxygen consumption $\left(\mathrm{VO}_{2}\right)$, minute ventilation $\left(\mathrm{V}_{\mathrm{E}}\right)$, and ventilatory equivalent for oxygen (VE/ $\mathrm{VO}_{2}$ ) were done at each three complete respiratory cycle. The $\mathrm{VO}_{2}$ mask and equipment were fastened to the subject after being positioned for the submaximal exercise. A face mask (Hans Rudolph V MaskTM, Shawnee, OH, USA) covered the mouth and nose, was attached to a bidirectional digital flowmeter and fastened to the subject with a mesh hairnet and Velcro straps. To establish a resting state, the following variables were considered: resting $\mathrm{VO}_{2}$ at 3.5 $\mathrm{ml} / \mathrm{kg} / \mathrm{min}-1$ and minute ventilation (VE) between 8 and $15 \mathrm{l} / \mathrm{min} .{ }^{14}$ Before each test, the gas analyzer system was calibrated, according to the manufacturer guidelines. 


\section{ECG recording and $\mathrm{HRV}$ analysis}

ECG was continuously recorded at a sampling rate of $1 \mathrm{kHz}$ with 12-bit resolution using a 12-lead ECG monitor system (CONTEC, model 8000D, New York, USA). All data were stored and analyzed offline. Heart rate and the R-R time series were extracted from 10-min ECG tracings (V5 lead), recorded at rest and after submaximal exercise testing using an R-R detection algorithm (MATLAB 7.0, The MathWorks, Inc, Natick, MA, USA). ECG recording occurred under spontaneous voluntary ventilation, at rest (average breathing rate of 10 breaths/min in the VP and of 16 breaths/min in the RAT) and after submaximal exercise testing (average breathing rate of 21 breaths/ $\mathrm{min}$ in the VP and 30 breaths/min in the RAT). The ECG tracing was manually inspected and edited for any ectopic beats. Both the ectopic and the post-extra-systolic beats were deleted and replaced by interpolated adjacent R-R interval values [12]. The following time domain of HRV variables were analyzed: $\mathrm{R}-\mathrm{R}$, the mean of all normal R-R intervals during the 10-min recording; SDNN, the standard deviation of normal R-R intervals; NN50, the number of interval differences of successive $\mathrm{NN}$ intervals greater than $50 \mathrm{~ms}$; pNN50, the percentage of normal R-R intervals that differ by more than $50 \mathrm{~ms}$; and RMSSD, the square root of the mean of the mean squared differences of successive R-R intervals. ${ }^{12}$

For frequency-domain analysis, R-R intervals time series were resampled to equal intervals by spline cubic interpolation method at $2 \mathrm{~Hz}$, and data were detrended by removing the mean value and the linear trend. Fast Fourier transformation was used for calculating the power spectrum (Welch's periodogram was employed to assess the 1024-point spectra with a Hanning window and 50\% overlap). PSD was obtained by integrating the power spectrum density function in the very low-frequency (VLF: 0.0033 and $0.04 \mathrm{~Hz}$.), the low-frequency (LF: $0.04-0.15 \mathrm{~Hz}$ ), and the high-frequency (HF: $0.15-0.40 \mathrm{~Hz}$ ) bands. The spectral power was also computed in normalized units for the HF $[\mathrm{HFnu}=\mathrm{HF}$ / (total power $-\mathrm{VLF}$ ) $\mathrm{x}$ 100] and LF [LFnu $=\mathrm{LF} /$ (total power - VLF) $\times 100]$, and the autonomic balance evaluated by the LF/HF. ${ }^{12}$

\section{Statistical Analysis}

All data is presented as mean \pm standard deviation. The data were initially analyzed using the Shapiro-Wilk normality test and the homoscedasticity test (Bartlett criterion). All variables showed normal distribution and homocedasticity. Intergroup differences were analyzed by two-way ANOVA, with post hoc comparisons (Bonferroni test) for autonomic modulation, $\mathrm{V}_{\mathrm{E}}, \mathrm{VO}_{2}$ and $\mathrm{V}_{\mathrm{E}} / \mathrm{VO}_{2}$. All the statistical analysis was performed with the Prism software (v. 5.0, Graphpad) and considered the significance level value $(\mathrm{p} \leq 0.05)$.

\section{Results}

The intraclass correlation coefficient was: RR interval: 0.92; r-MSSD: 0.89; LF: 0.94; TP: 0.95; HF: 0.93; LF/HF: $0.94 ; \mathrm{VO}_{2}: 0.96 ; \mathrm{V}_{\mathrm{E}} / \mathrm{VO}_{2}: 0.92$; and $\mathrm{V}_{\mathrm{E}}: 0.94$.

The autonomic cardiac behavior in relation to inter-group analysis (VP vs. RAT) revealed significant differences at rest for RR interval and RMSSD $(\mathrm{p}<0.01)$ when analyzed in the time domain (Table 1). However, when analyzing the frequency domain

Table 1: Time-domain and Frequency-domian of HRV at rest and post-effort.

\begin{tabular}{lcccc}
\hline & Rest RAT & Rest VP & Post-effort RAT & Post-effort VP \\
\hline RR interval $(\mathrm{ms})$ & $899,3 \pm 109,8$ & $1097 \pm 157,4^{*}$ & $726,3 \pm 89,09$ & $794,1 \pm 36,9$ \\
rMSSD $(\mathrm{ms})$ & $52,2 \pm 21,6$ & $74,7 \pm 26,2^{\star}$ & $19,7 \pm 6,8$ & $29,8 \pm 8,1$ \\
Total Power $\left(\mathrm{ms}^{2}\right)$ & $3560 \pm 2233$ & $6308 \pm 2654^{\star *}$ & $1147 \pm 661,7$ & $2146 \pm 983,1$ \\
LF $\left(\mathrm{ms}^{2}\right)$ & $1154 \pm 724,4$ & $2609 \pm 1323^{\star \star}$ & $476,9 \pm 254,5$ & $1015 \pm 533,2$ \\
HF $\left(\mathrm{ms}^{2}\right)$ & $1082 \pm 728,7$ & $1416 \pm 688,2$ & $134,6 \pm 98,5$ & $287 \pm 170,5$ \\
LF/HF ratio (\%) & $1,6 \pm 1,4$ & $2,2 \pm 1,7$ & $3,9 \pm 1,3$ & $3,7 \pm 1,3$ \\
\hline
\end{tabular}

Values are expressed as mean \pm SEM. RAT $=$ recreationally aerobic trained; VP $=$ athletes.

${ }^{*} p<0.01 ;-$ rest VP vs. rest RAT. ** $p<0.001$ - rest VP vs. rest RAT. 
at rest, the VP showed a significant increase in Total power and LF ( $\mathrm{p}<0.001)$ when compared to RAT (Table 1). However, the variables $\mathrm{HF}$ and $\mathrm{LF} / \mathrm{HF}$ ratio showed no significant difference between groups. No significant difference was demonstrated between groups in the post-effort referring to the variables of time domain and frequency HRV ( $\mathrm{p}>0.05)$.

Moreover, the $\mathrm{VO}_{2}$ submaximal, the ventilatory equivalent to $\mathrm{V}_{\mathrm{E}} / \mathrm{VO}_{2}$ and $\mathrm{V}_{\mathrm{E}}$ did not exhibit significant differences between VP vs. RAT at pre-exercise and peak of exercise, or five minutes after submaximal test (Figure 1A, 1B and 1C; $>>0.05$ ).

\section{Discussion}

Few studies have compared HRV response and exercise between groups of subjects with different levels of aerobic training. Similar HRV measures were reported for athletes during exercise at 50\% maximum workload $^{8}$ and during recumbent cycling at $20 \%, 40 \%$ and $50 \%$ to $\mathrm{VO} 2 \mathrm{max} .{ }^{27}$ The results obtained in the present study related to the variables over time (mean RR and RMSSD) and frequency domain (TP and LF) was significantly higher in VP when compared to RAT. It is possible that these results are directly correlated to changes in neural mechanisms of cardiac autonomic control triggered by successive anaerobic stimuli under which volleyball athletes are exposed to daily during sports preparation. These adaptations may be associated to the developed level of physical capacity of individuals engaged in a systematized and periodized aerobic and anaerobic training program. ${ }^{8,16,17,22}$

Some authors suggest that the increase of physical capacity acquired by training can result in signifi-

(A)

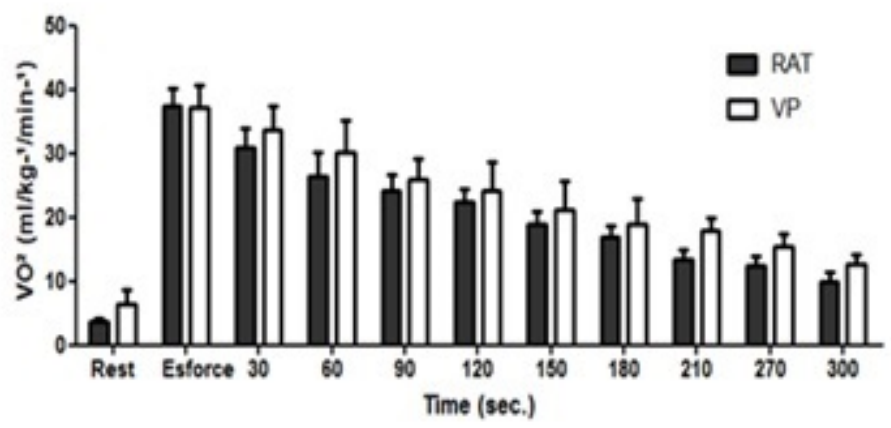

(B)

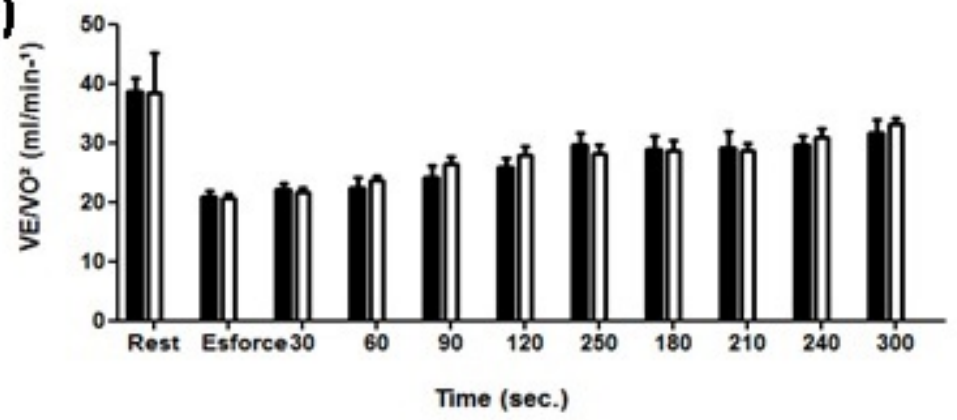

(C)

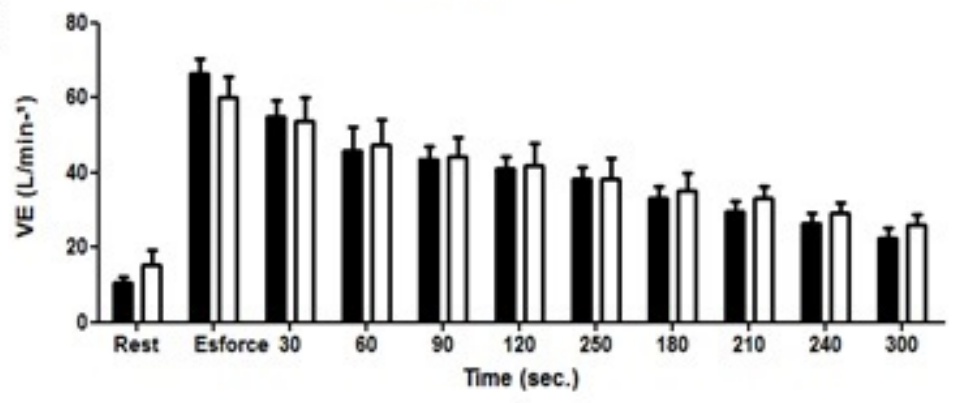

Figure 1. Mean respired gas analysis at rest and after cessation of exercise testing, following VP and RAT. VP: volleyball players. RAT: recreational aerobically trained individuals. 
cant changes to the neural mechanisms of autonomic cardiac control. ${ }^{2,8,24}$ These changes are directly associated with an increase of vagal activity and possible reduction in sympathetic activity, both at rest and in the initial phase of exercise. ${ }^{2,13,24,35}$ However, individuals in training showed smaller increases in $\mathrm{HR}$ at the beginning of the stress test and also exhibited a smaller HR in submaximal exercise. ${ }^{9,27}$ Immediately following post-exercise, individuals with high levels of physical capacity seem to also show a marked vagal reactivity and a smaller increase in sympathetic drive to the heart. ${ }^{3,21,29,31}$ These added features are adjustments/adaptations triggered by physical training and favor less myocardial work in different situations (at rest and during/recovery training). ${ }^{3}$

Our findings showed no significant difference between groups (VP vs. RAT) in the pre and postexercise phases when markers of behavior vagal (HF and $\mathrm{LF} / \mathrm{HF}$ ) were investigated and it is in accordance with our previous pilot study. ${ }^{30}$ This result may be related to oxygen consumption at peak exercise, as both groups were characterized by physical activity. This hypothesis corroborates with current scientific literature on how the methodological variables involved in physical training, such as intensity and volume, can interfere with autonomic responses and resistance/tolerance to exercise. ${ }^{21,27,31,36}$ In a study to determine whether training intensity would affect HRV, two groups of middle-aged men were trained for 20 weeks. One of the groups performed exercise at $55 \% \mathrm{VO}_{2 \max }$, while the other group performed exercise at $75 \% \mathrm{VO}_{2 \max }$. Both groups trained on average four times per week, for 33 minutes per session. After 20 weeks no differences were found in the time and frequency domain variables of HRV for either group. ${ }^{37}$

Endurance athletes have higher vagal activity at rest when compared to sedentary individuals and also a rapid recovery of HR. ${ }^{21,28}$ In another study, elite cross-country athletes had a higher parasympathetic activity when compared to the physically active and sedentary population. ${ }^{7}$ In present study, VP group demonstrated higher vagal activity (RMSSD index) at rest when compared to RAT. The RMSSD has better statistical properties for assessment of parasympathetic activity, uncontaminated by sympathetically mediated HRV. ${ }^{12}$ The results suggests that the volume, type of stimulus and specific characteristics of the sport may induces sinus bradycardia in resting conditions, and a slower increase in heart rate at any degree of submaximal oxygen uptake due to a shift of the sympathovagal balance towards parasympathetic dominance., ${ }^{9,25}$ Thus, the exercise program might be an important factor when looking at the effects of exercise training on HRV.

Total power consists in the sum of frequency bands ranging from 0 to $0.5 \mathrm{~Hz}$ and has also been found to indicate mainly vagal activity fluctuations at rest. ${ }^{2,12}$ The present findings reveal a significant increase in the TP of HRV in athletes at rest. Possibly, because athletes showed larger end-diastolic volumes and a lower minimum heart rate when compared to RAT. ${ }^{38}$ After exercise stress, it appears that the major percentage of TP operates predominantly in the LF band, and therefore, would be a possible indicator of changes in total autonomic modulation instead of only changes in the modulation vagal. ${ }^{2,12,38}$ The LF may reflect multiple mechanisms of cardiac autonomic control, such as the swing arm of sympathetic/parasympathetic and baroreceptor reflex response. Due to the results of this study, it is speculated that at rest, the increase in LF athletes may be related to changes in cardio-vagal activity present in the fluctuations of RR intervals in the active band LF. ${ }^{24,35}$ This way, eliciting atrial and ventricular dilation, would induce intrinsic electrophysiological adaptations and enhance parasympathetic activity ${ }^{39}$. Another factor that could also influence the elevation of the LF component in athletes would be breathing rate. When their breathing reached 9 breaths/min, they became active in the area of LF $(0.04 \mathrm{~Hz}$ to $0.15 \mathrm{~Hz})$. However, individuals kept the variation of respiratory tracts in the range of 12 to 18 breaths/min, showing its activity in HF $(0.2 \mathrm{~Hz}$ to $0.3 \mathrm{~Hz}){ }^{23}$

It would be necessary to elucidate the results and the use of a maximal exercise test protocol, which is crucial for establishing anaerobic threshold and $\mathrm{VO}_{2 \max }$. This University follows the position supported by the American Heart Association, which requires the presence of a doctor for an exertion test of maximal effort, a factor that could not be accomplished. Also, the number of volunteers for this study sample was limited due to the incompatibility of the sports calendar.

\section{Conclusions}

Recreational aerobically trained individuals and professional volleyball players showed a different response of the training-related increase in vagal tone. 
The variables such as frequency, intensity and volume may to interfere the autonomic cardiac modulation and possibly indicating a superior protection against exercise-induced ventricular arrhythmias and lower risk of sudden cardiac deaths.

\section{Acknowledgments}

PhD. Roberto Simão and $\mathrm{PhD}$. Alex Souto Maior would like to thank the Research and Development Foundation of Rio de Janeiro State (FAPERJ) for the research grant support.

Declaration of interest

The authors have no conflicts of interest to declare.

\section{References}

1. Berntson GG, Bigger JT Jr, Eckberg DL, Grossman $P$, Kaufmann PG, Malik M, et al. Heart rate variability: origins, methods, and interpretive caveats. Psychophysiology. 1997;34:623-48.

2. Eckberg DL. Sympathovagal balance. A critical appraisal. Circulation. 1997;96:3224-32.

3.Perini $R$, Veicsteinas A. Heart rate variability and autonomic activity at rest and during exercise in various physiological conditions. Eur J Appl Physiol. 2003; 90:317-25.

4. Holter NJ. New method for heart studies. Science. 1961; 134:1214-20.

5. American College of Sports Medicine. The recommended quantity and quality of exercise for developing and maintaining cardiorespiratory and muscular fitness, and flexibility in healthy adults. Med Sci Sports Exerc. 1998;30:975-91.

6. American College of Sports Medicine; American Heart Association. Exercise and acute cardiovascular events: placing the risks into perspective. Med Sci Sports Exerc. 2007; 39:886-97.

7. Berkoff DJ, Cairns CB, Sanchez LD, Moorman CT. Heart rate variability in elite American track-and-field athletes. J Strength Cond Res. 2007; 21:227-31.

8. Dixon EM, Kamath MV, McCartney N, Fallen EL. Neural regulation of heart rate variability in endurance athletes and sedentary controls. Cardiovasc Res. 1992;26:713-19.

9.Gregoire J, Tuck S, Yamamoto Y, Hughson RL. Heart rate variability at rest and exercise: influence of age, gender, and physical training. Can J Appl Physiol. 1996;21:455-447.

10.Sacknoff DM, Gleim GW, Stachenfeld N, Coplan NL. Effect of athletic training on heart rate variability. Am Heart J. 1994;127:1275-8.

11.Stickland MK, Miller JD. The best medicine: exercise training normalizes chemosensitivity and sympathoexcitation in heart failure. J Appl Physiol. 2008;105:779-81.

12. Task Force of the European Society of Cardiology and the North American Society of Pacing and Electrophysiology. Heart rate variability: standards of measurement, physiological interpretation and clinical use. Circulation. 1996;93:1043-65.

13. Goldberger JJ, Le FK, Lahiri M, Kannankeril PJ, Ng J, Kadish AH.Assessment of parasympathetic reactivation after exercise. Am J Physiol, Heart Circ Physiol. 2006;290:2446-52
14. Gibbons RJ, Balady GJ, Bricker JT, Chaitman BR, Fletcher GF, Froelicher VF, et al. ACC/AHA 2002 Guideline update for exercise testing: summary article. A report of the American College of Cardiology/American Heart Association Task Force on Practice Guidelines (Committee to Update the 1997 Exercise Testing Guidelines). J Am Coll Cardiol. 2002;40:153140.

15. Robbins M, Francis G, Pashkow FJ, Snader CE, Hoercher K, Young JB, et al. Ventilatory and heart rate responses to exercise: better predictors of heart failure mortality than peak oxygen consumption. Circulation. 1999;100:2411-17.

16. Bonaduce D, Petretta M, Cavallaro V, Apicella C, lanniciello $\mathrm{A}$, Romano $\mathrm{M}$, et al. Intensive training and cardiac autonomic control in high level athletes. Med Sci Sports Exerc. 1998;30:691-9.

17. Brown SJ, Brown JA. Resting and post-exercise cardiac autonomic control in trained master athletes. J Physiol Sci. 2007;57:23-9.

18.Kaikkonen P, Rusko H, Martinma K. Post-exercise heart rate variability of endurance athletes after different high-intensity exercise interventions. Scand J Med Sci Sports. 2008;18:51119.

19.Katona PG, McLean M, Dighton DH, Guz A. Sympathetic and parasympathetic cardiac control in athletes and nonathletes at rest. J Appl Physiol Respir, Environ Exerc Physiol. 1982;52:1652-7.

20.Ken-ichi I, Zhang R, Zuckerman JH, Levine BD. Dose-response relationship of the cardiovascular adaptation to endurance training in healthy adults: how much training for what benefit? J Appl Physiol. 2003;95:1575-83.

21.Shin K, Minamitani H, Onishi S. Autonomic differences between athletes and non athletes: spectral analysis approach. Med Sci Sports Exerc. 1997;29:1482-90.

22.Borresen J, Lambert MI. Autonomic control of heart rate during and after exercise: measurements and implications for monitoring training status. Sports Med. 2008;38:633-46.

23. Brown TE, Beightol LA, Koh J, Eckberg DL.. Important influence of respiration on human R-R interval power spectra is largely ignored. J Appl Physiol. 1993;75:2310-17.

24. Furlan R, Piazza S, Dell'Orto S, Gentile E, Cerutti S, Pagani $M$, et al. Early and late effects of exercise and athletic training on neural mechanisms controlling heart rate. Cardiovasc Res. 1993;27:482-8.

25. Karvonen MJ, Kentala E, Mustala O. The effects of training on heart rate: a longitudinal study. Ann Med Exp Biol Fenn. 1957;35:307-15.

26. Mourot L, Bouhaddi M, Tordi N, Rouillon JD, Regnard J. Shortand long-term effects of a single bout of exercise on heart rate variability: comparison between constant and interval training exercises. Eur J Appl Physiol. 2004;92:508-17.

27. Perini R, Fisher N, Veicsteinas A, Pendergast DR. Aerobic training and cardiovascular responses at rest and during exercise in older men and women. Med Sci Sports Exerc. 2002;34:700-8.

28. Sztajzel J, Jung M, Sievert K, Bayes De Luna A.. Cardiac autonomic profile in different sports disciplines during all-day activity. J Sports Med. Phys Fit.2008;48:495-501.

29. Thompson PD, Crouse SF, Goodpaster B, Kelley D, Moyna $\mathrm{N}$, Pescatello $\mathrm{L}$. The acute versus the chronic response to exercise. Med Sci Sports Exerc. 2001;33:438-45.

30. Menezes PR, Simão R, Neto SEM, Maior AS. Autonomic cardiac and cardiorespiratory reponses in volleyball athletes compared to trained individuals. REV SOCERJ. 2009;22:235-42. 
31. Seiler S, Haugen O, Kuffel E. Autonomic recovery after exercise in trained athletes: intensity and duration effects. Med Sci Sports Exerc. 2007;39:1366-73.

32. Mazon J, Gastaldi A, Di Sacco T, Cozza I, Dutra S, Souza H.. Effects of training periodization on cardiac autonomic modulation and endogenous stress markers in volleyball players. Scand J Med Sci Sports. 2013;23:114-20.

33. Fletcher GF, Balady GJ, Amsterdam EA, Chaitman B, Eckel $\mathrm{R}$, Fleg J. Exercise standards for testing and training: a statement for healthcare professionals from the American Heart Association. Circulation. 2001;104:1694-1640.

34. Craig CL, Marshall AL, Sjöström M, Bauman AE, Booth ML, Ainsworth BE. International physical activity questionnaire: 12-country reliability and validity. Med Sci Sports Exerc. 2003;35:1381-95.
35. Dabire H, Mestivier D, Jarnet J, Safar ME, Chau NP.. Quantification of sympathetic and parasympathetic tones by nonlinear indexes in normotensive rats. Am J Physiol. 1998; 275:1290-7.

36. Shin K, Minamitani H, Onishi S, Yamazaki H, Lee M.. The power spectral analysis of heart rate variability in athletes during dynamic exercise. Part I. Clin Cardiol. 1995;18:583-6.

37. Loimaala A, Huikuri H, Oja P. Controlled 5-mo aerobic training improves heart rate but not heart rate variability or baroreflex sensitivity. J Appl Physiol. 2000;89:1825-9.

38. Fürholz M, Radtke T, Roten L, Tanner H, Wilhelm I, Schmid $\mathrm{J}$, et al. Training-related modulations of the autonomic nervous system in endurance athletes: is female gender cardioprotective?. Eur J Appl Physiol. 2013;113:631-40.

39. Aubert A, Seps B, Beckers F. Heart Rate Variability in Athletes. Sports Med. 2003; 33: 889-919. 